ARTICLE

\title{
The hereditary mutation G51D unlocks a distinct fibril strain transmissible to wild-type $\alpha$-synuclein
}

\author{
Yunpeng Sun (1) 1,2,6, Houfang Long (1) 1,2,6, Wencheng Xia ${ }^{1,2}$, Kun Wang ${ }^{1,2}$, Xia Zhang ${ }^{3}$, Bo Sun (1) ${ }^{3}$, Qin Cao ${ }^{4}$, \\ Yaoyang Zhang (iD) ${ }^{1,2}$, Bin Dai ${ }^{5}$, Dan Li (i] ${ }^{4}$ \& Cong Liu (1) ${ }^{1,2 凶}$
}

$\alpha$-Synuclein ( $\alpha$-Syn) can form different fibril strains with distinct polymorphs and neuropathologies, which is associated with the clinicopathological variability in synucleinopathies. How different $\alpha$-syn fibril strains are produced and selected under disease conditions remains poorly understood. In this study, we show that the hereditary mutation G51D induces $\alpha$-syn to form a distinct fibril strain in vitro. The cryogenic electron microscopy (cryo-EM) structure of the G51D fibril strain was determined at $2.96 \AA$ resolution. The G51D fibril displays a relatively small and extended serpentine fold distinct from other $\alpha$-syn fibril structures. Moreover, we show by cryo-EM that wild-type (WT) $\alpha$-syn can assembly into the G51D fibril strain via cross-seeding with G51D fibrils. Our study reveals a distinct structure of G51D fibril strain triggered by G51D mutation but feasibly adopted by both WT and G51D $\alpha$-syn, which suggests the cross-seeding and strain selection of WT and mutant $\alpha$-syn in familial Parkinson's disease (fPD).

\footnotetext{
${ }^{1}$ Interdisciplinary Research Center on Biology and Chemistry, Shanghai Institute of Organic Chemistry, Chinese Academy of Sciences, 201210 Shanghai, China. ${ }^{2}$ University of Chinese Academy of Sciences, 100049 Beijing, China. ${ }^{3}$ School of Life Science and Technology, ShanghaiTech University, 201210 Shanghai, China. ${ }^{4}$ Bio-X Institutes, Key Laboratory for the Genetics of Developmental and Neuropsychiatric Disorders, Ministry of Education, Shanghai Jiao Tong University, 200030 Shanghai, China. ${ }^{5}$ Institute of Nano Biomedicine and Engineering, Department of Instrument Science and Engineering, School of Electronic Information and Electrical Engineering, Shanghai Jiao Tong University, 200210 Shanghai, China. ${ }^{6}$ These authors contributed equally: Yunpeng Sun, Houfang Long. ${ }^{凶}$ email: liulab@sioc.ac.cn
} 
-Syn is a highly abundant presynaptic protein in neurons. It forms amyloid aggregation and condenses into Lewy bodies (LB) and Lewy neurites (LN) which are histological hallmarks of Parkinson's disease (PD) and other synucleinopathies ${ }^{1-3}$. Mounting evidence has shown that amyloid fibrils of $\alpha$-syn serve as a key pathological entity that may impair protein homeostasis, sequester mitochondria, and mediate cellto-cell transmission and spread of the $\alpha$-syn pathology during the disease progression ${ }^{4-7}$. Strikingly, $\alpha$-syn can form various fibril strains both in vitro and in vivo ${ }^{8-14}$. Different $\alpha$-syn fibril strains exhibit distinct neuropathologies. Therefore, different strains may be involved in different types of synucleinopathies with a wide spectrum of heterogeneous clinical symptoms ${ }^{14-18}$, including PD, multiple system atrophy (MSA), dementia with Lewy bodies (DLB) ${ }^{17,19,20}$. Indeed, the $\alpha$-syn fibrils derived from the brains or the cerebrospinal fluid (CSF) of PD and MSA patients showed not only different fibril polymorphs (strains) but also distinct pathological properties such as cell-to-cell propagation and neurotoxicity $15,17,21$. Thus, it is important to understand how a-syn selects to adopt one fibril strain but not the other under certain circumstances.

Recent cryo-EM studies have shown that hereditary mutations (e.g., H50Q, E46K, and A53T) and disease-associated phosphorylation (pY39) of $\alpha$-syn can induce distinct fibril strains ${ }^{22-26}$. This implies that mutation and post-translational modification (PTM) may play important roles in $\alpha$-syn strain selection. In this study, we focused on the hereditary mutation G51D which is one of the eight disease-causing mutations identified in fPD and explored its role in $\alpha$-syn fibril strain formation and selection. G51D mutation was firstly described from a French family with a Parkinsonian-pyramidal syndrome ${ }^{27}$. An unusual PD phenotype of G51D was characterized by early disease onset, rapid progression, and death within a few years ${ }^{27,28}$. Previous studies showed that G51D a-syn fibrils are more toxic than WT a-syn fibrils in cell models ${ }^{29,30}$. Intriguingly, gene $S N C A$ of the fPD patients with G51D mutation are heterozygous ${ }^{27}$, so both WT and G51D a-syn co-exist in the patients. Therefore, it is important to investigate whether and how the G51D and WT a-syn may influence the fibril strain selection of each other.

In this work, we determined the cryo-EM structure of G51D fibril strain prepared in vitro at a resolution of $2.96 \AA$. The G51D strain displays potent neuropathology and exhibits a distinct structure from all known a-syn strains. Moreover, we found that the G51D strain can induce WT $\alpha$-syn monomer to form amyloid fibril with a similar structure to the G51D strain via cross-seeding. Our work demonstrates that the G51D a-syn fibril structure presented here can be adopted by both G51D and WT a-syn, which indicates that the mutant and WT $\alpha$-syn may concertedly accelerate the disease progression in $\mathrm{PD}$ with G51D mutation.

\section{Results}

G51D a-syn forms neuropathological fibrils with a righthanded helical twist. To investigate how G51D mutation influences the fibril formation of $\alpha$-syn, we overexpressed and purified full-length G51D $\alpha$-syn with N-terminal acetylated. By performing the Thioflavin $\mathrm{T}$ (ThT) kinetic assay and negativestaining transmission electron microscopy (NS-TEM), we showed that G51D a-syn spontaneously forms long and unbranched amyloid fibrils with a lag time of $\sim 16 \mathrm{~h}$ in $50 \mathrm{mM}$ phosphate buffer, pH 7.0, $50 \mathrm{mM} \mathrm{NaCl}$ (referred to as PB buffer) (Fig. 1a). The G51D fibrils are of good homogeneity with half pitches in the range of $67.0 \pm 9.6 \mathrm{~nm}$ (Fig. 1b). We confirmed that the G51D fibrils are formed by full-length G51D $\alpha$-syn by SDS-PAGE, Western blot, and matrix-assisted laser desorption/ionization time-of-flight mass spectrometry (MALDI-TOF MS)
(Supplementary Fig. 1). In the presence of preformed fibrils (PFFs) of G51D a-syn, the G51D a-syn monomer rapidly forms fibril with greatly shortened lag time and reaches a plateau in $55 \mathrm{~h}$ (Fig. 1a). The atomic force microscopy (AFM) further reveals that the G51D fibril features a right-handed helical twist (Fig. 1c and Supplementary Fig. 2), which is distinct from all known a-syn fibrils (left-handed) except for the E46K fibril ${ }^{9-14,22-26}$. To further confirm the causative role of G51D mutation in the structural change, we prepared fibrils with WT a-syn under the same condition (i.e., in the PB buffer). AFM and NS-TEM images showed that WT a-syn formed a mixture of three polymorphs including straight polymorph $(\sim 93 \%)$, and two left-handed twist polymorphs ( $\sim 3.6 \%$ and $\sim 2.6 \%$, respectively) in PB buffer, which are all distinct from the right-handed twist morphology of the G51D fibril formed under the same condition (Supplementary Fig. 2).

We next sought to explore the physiochemical and pathological properties of the G51D fibrils and compared it with a previously well-characterized fibril polymorph formed by WT $\alpha$-syn (referred to as $\left.\mathrm{WT}_{1 \mathrm{a}}\right)^{11}$. We firstly measured the fibril stability by using a proteinase $\mathrm{K}(\mathrm{PK})$ digestion assay. The result showed that the G51D PFFs are digested markedly faster than the $\mathrm{WT}_{1 \mathrm{a}}$ PFFs (Supplementary Fig. 3a). We further investigated the fibril stability by sonication. The NS-TEM images showed that the G51D fibrils were fragmented into shorter pieces than the $\mathrm{WT}_{1 \mathrm{a}}$ fibrils upon sonication (Supplementary Fig. 3b). Moreover, the cellular viability results showed that the G51D PFFs exhibit stronger cellular toxicity to SH-SY5Y cells than the $\mathrm{WT}_{1 \mathrm{a}}$ PFFs (Supplementary Fig. 4a). Finally, we examined the neuronal propagation capability of G51D by using a well-established primary neuron assay ${ }^{31}$. Notably, the G51D PFFs induced significantly more pathological aggregation of a-syn in neurons than the $\mathrm{WT}_{1 \mathrm{a}}$ PFFs (Supplementary Fig. 4b, c). Taken together, these data indicate that the G51D fibrils prepared in this work display a distinct fibril polymorph with decreased stability and potent neuropathology.

Cryo-EM structure determination of the G51D $\alpha$-syn fibril. We next sought to determine the atomic structure of the G51D fibril by using cryo-EM. We collected 1869 micrographs from a glowdischarged holey carbon grid containing well-dispersed G51D fibrils. One major fibril polymorph was identified with a population of over $95 \%$ of the total 88,917 fibril segments with a 1024 pixel box size from the two-dimensional (2D) classification. Based on the high-quality cryo-EM density map acquired, we managed to build a structural model of G51D fibril with an overall resolution of $2.96 \AA$ (Supplementary Fig. 5). The G51D fibril is composed of two intertwining protofilaments with a right-handed helix (Fig. 2a). The subunits within each protofilament stack along the fibril axis with a helical twist of $1.26^{\circ}$ and helical rise of $4.86 \mathrm{~A}$ (Fig. 2a and Table 1). Residues 50-98 of $\alpha$-syn form the fibril core (FC), and the rest of the residues in the $\mathrm{N}$ - and $\mathrm{C}$-terminal regions are relatively flexible in the fibril and invisible by cryo-EM imaging (Fig. 2b). Residues 74-79 from opposing asyn subunits form a highly complementary and dry interface for zippering the two protofilaments together (Fig. 2c).

Structure comparison of WT, G51D, and E46K $\alpha$-syn fibrils. The G51D FC consists of six $\beta$-strands $\left(\beta_{1}-\beta_{6}\right)$ which arranges into an extended serpentine fold (Fig. $2 \mathrm{~d}$ ). The overall structure of G51D fibril is distinct from all the 19 previously determined $\alpha$ syn fibril structures formed by either WT a-syn or variants $9-14,22-26$, although the G51D fibril exhibits structural similarity with WT polymorph 1a $\left(\mathrm{WT}_{1 \mathrm{a}}, \mathrm{PDB}\right.$ ID: $\left.6 \mathrm{~A} 6 \mathrm{~B}\right)$ and E46K fibril (PDB ID: 6L4S) ${ }^{9,25}$ (Fig. 3a). In particular, residues 

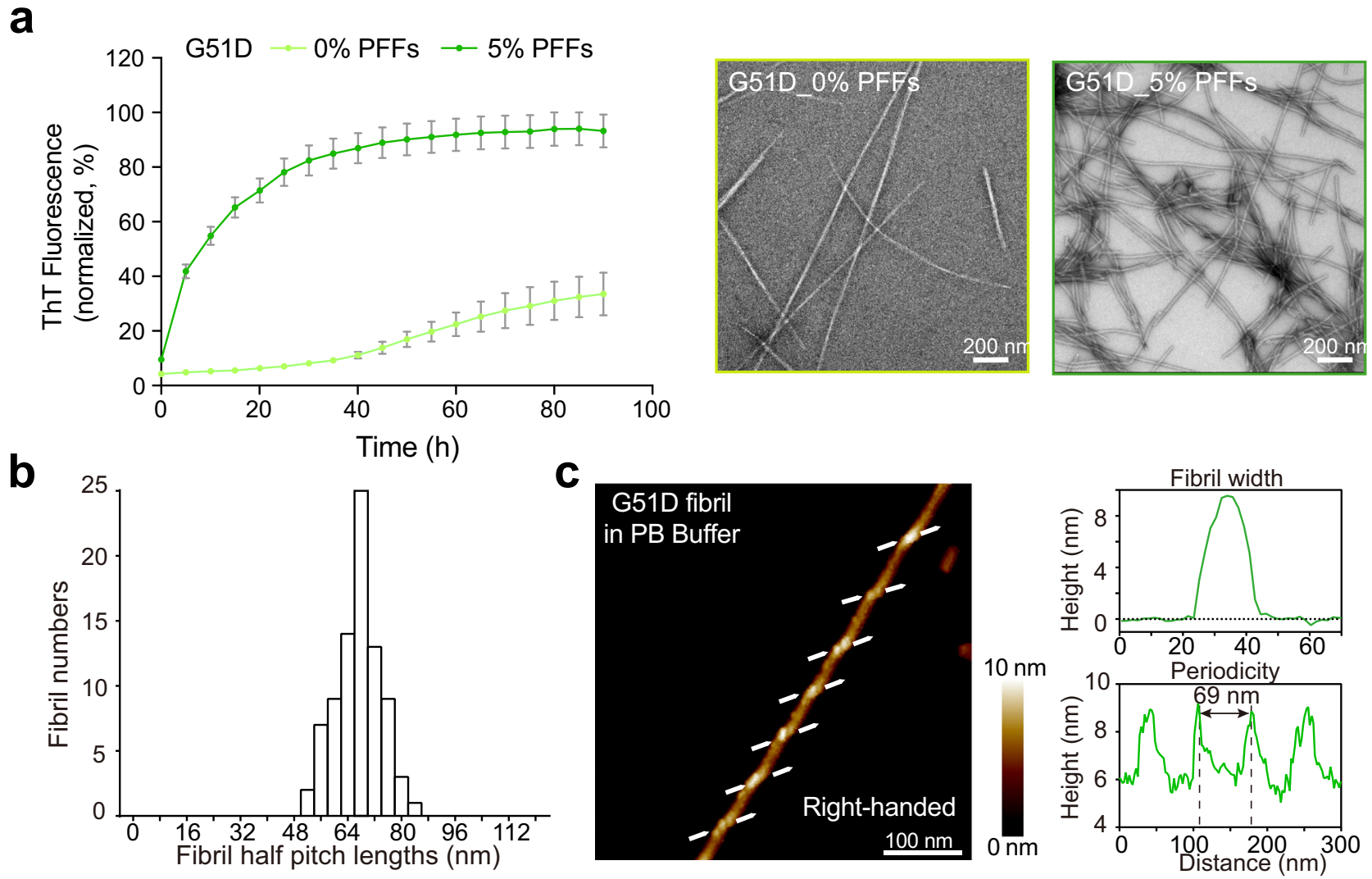

Fig. 1 Characterization of the fibrils formed by G51D $\boldsymbol{\alpha}$-syn. a ThT kinetic assay of the G51D fibril formation with (colored in dark green) and without PFFs ( 5 mol\%) (colored in light green) in PB Buffer ( $50 \mathrm{mM}$ phosphate buffer, $\mathrm{pH} 7.0,50 \mathrm{mM} \mathrm{NaCl}$ ). Data are shown as mean $\pm \mathrm{s} . \mathrm{d} ., n=5$ independent samples (left panel). The fibrils formed at the endpoint of the ThT kinetic assay were characterized by NS-TEM (right panel). Scale bar: $200 \mathrm{~nm}$. b Histogram of half-pitch lengths of G51D fibrils (0\% PFFs) measured from NS-TEM images $(n=83)$. c AFM image of a G51D fibril. The arrows at both sides of the fibril indicate the starting points of the fibril protrusions to clarify the handedness. Images were acquired with a $1.45 \mathrm{~Hz}$ rate at $512 \times 512$ pixels. Fibrils formed by G51D in >3 independent experiments provide reproducible images. The fibril width and periodicity were analyzed by the Nanoscope software.

60-98 form a similar architecture in G51D, $\mathrm{WT}_{1 \mathrm{a}}$, and $\mathrm{E} 46 \mathrm{~K}$ fibrils (Fig. 3a). However, both G51D and E46K fibrils feature a smaller and more loose FC than that of the $\mathrm{WT}_{1 \mathrm{a}}$ (Fig. 3a, b). In the $\mathrm{WT}_{1 \mathrm{a}}$ structure, G51 is involved in the steric-zipper interface of the two protofilaments formed by residues 50-57 $\left({ }^{50}\right.$ HGVATVAE $\left.^{57}\right)$, which is assumed to be disrupted by G51D mutation (Fig. 3b, c). Instead, this segment with G51D mutation flips $180^{\circ}$ perpendicular to the fibril axis and forms a $\beta$-hairpin structure with residues $60-66$ in the mutant fibril (Fig. 3d). Meanwhile, V74, A76, and A78 that used to be buried inside the $\mathrm{WT}_{1 \mathrm{a}} \mathrm{FC}$ are exposed in G51D FC and form a homotypical stericzipper interface to bundle the two protofilaments together (Fig. 3b, c).

In the E46K fibril structure, residues $45-53$ form an additional $\beta$-turn, which attaches to the $\beta$-hairpin by a salt bridge formed by E57 and K45. G51 is essential in forming the $\beta$-turn (Fig. 3e). Thus, the replacement of Gly to Asp disrupts the $\beta$-turn formation and leaves the entire N-terminal (residues 1-49) flexible and invisible from cryo-EM (Fig. 3b, e). Therefore, despite that G51D FC shares structural similarity with $\mathrm{WT}_{1 \mathrm{a}}$ and $\mathrm{E} 46 \mathrm{~K}$ FCs, G51D mutation excludes the formation of either $\mathrm{WT}_{1 \mathrm{a}}$ or E46K fibril structures.

WT $\alpha$-syn forms G51D fibril strain by cross-seeding. Since both WT and G51D a-syn co-exist in the fPD patients carrying heterozygous G51D mutation ${ }^{27}$, we next asked whether WT a-syn can form the G51D fibril strain in the presence of the preformed G51D fibril PFFs (Fig. 4a). As previously reported, WT a-syn forms left-handed fibrils featuring $\mathrm{WT}_{1 \mathrm{a}}$ structure under the

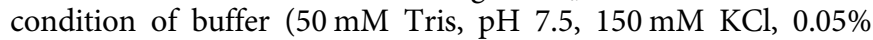
$\left.\mathrm{NaN}_{3}\right)^{11}$. Under the same fibrillation condition, we examined the seeding effect of $\mathrm{WT}_{1 \mathrm{a}}$ PFFs and G51D PFFs for WT a-syn fibrillation, respectively. The result showed that both WT and G51D PFFs exhibit potent seeding activity in inducing fibrillation of WT a-syn monomer monitored by NS-TEM and the ThT kinetics assay (Fig. 4b). Strikingly, AFM measurement revealed that the WT a-syn fibril cross-seeded by G51D PFFs (termed as $\mathrm{WT}_{51 \mathrm{cs}}$ fibril) features a right-handed twist same as its template G51D fibril (Fig. 4c). While the fibril seeded by $\mathrm{WT}_{1 \mathrm{a}}$ PFFs (termed as $\mathrm{WT}_{\text {wts }}$ fibril) displays a left-handed twist and inherited the fibril morphology of its template-WT $\mathrm{WT}_{1 \mathrm{a}}$ fibril (Fig. $4 \mathrm{~d}$ and Supplementary Fig. 6).

We further explored the structures of the $\mathrm{WT}_{51 \mathrm{cs}}$ and $\mathrm{WT}_{\mathrm{wts}}$ fibrils by using cryo-EM (Fig. 5a-d). We fixed the $\mathrm{WT}_{51 \mathrm{cs}}$ and $\mathrm{WT}_{\text {wts }}$ fibril samples on the carbon grids in liquid ethane and collected 725 and 1063 cryo-EM micrographs, respectively. 2D classifications showed one dominant species of the $\mathrm{WT}_{51 \mathrm{cs}}$ fibril sample with a population of $\sim 97 \%$ of the total 44,870 fibril segments with 1200-pixel box size. $\mathrm{WT}_{\mathrm{wts}}$ fibril sample contains the main species with a population of $\sim 91 \%$ of the total 44,909 fibril segments with a 1024-pixel box size. Notably, three-dimensional (3D) reconstructions revealed that $\mathrm{WT}_{51 \mathrm{cs}}$ exhibits a highly similar $\mathrm{FC}$ structure as that of the G51D fibril, indicating that the structure of G51D fibril is inherited by WT a-syn upon cross-seeding (Fig. $5 \mathrm{c}, \mathrm{e}$ ). Of note, compared to $\mathrm{G} 51 \mathrm{D}, \mathrm{WT}_{51 \mathrm{cs}}$ showed additional electron density adjacent to the $\beta$-hairpin (residues 51-66) (Fig. 5e). 

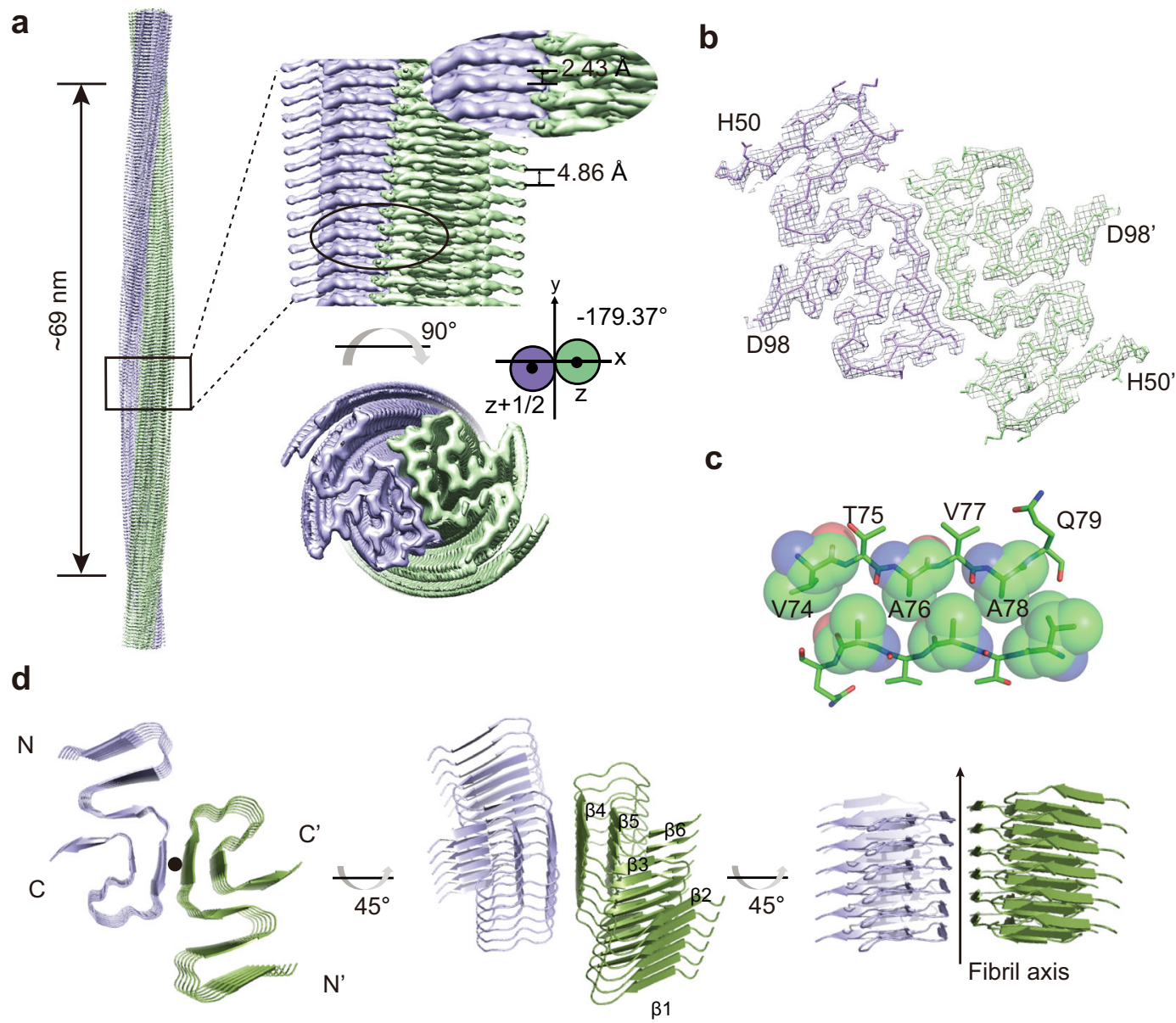

Fig. 2 Cryo-EM structure of the G51D $\boldsymbol{\alpha}$-syn fibril. a Cryo-EM density map of G51D $\alpha$-syn fibril. Fibril parameters including half-pitch, twist angle, and rise are marked. The two protofilaments are colored in medium purple and forest green, respectively. The map was prepared by UCSF Chimera v1.13. b Crosssection view of the density map with a built-in structure model. c The protofilament interface of G51D fibril is shown in sticks. Residues involved in the inter-protofilament interactions are indicated in spheres. $\mathbf{d}$ Views of six layers of G51D $\alpha$-syn fibrils are shown in the cartoon. The fibril axis is indicated. The $\beta$-strands are numbered and labeled accordingly.

The density may represent the $\beta$-turn consisting of residues $45-53$ as observed in the $\mathrm{E} 46 \mathrm{~K}$ strain. As for the $\mathrm{WT}_{\mathrm{wts}}$ fibril, $3 \mathrm{D}$ reconstructions confirmed that $\mathrm{WT}_{\mathrm{wts}}$ recapitulates the architecture of its template $\mathrm{WT}_{1 \mathrm{a}}$ fibril (Fig. 5d, f). Taken together, our results demonstrate that G51D mutation is sufficient but not necessary for forming the structure of the G51D fibril strain. WT $\alpha$-syn can also adopt the G51D-like fibril structure in the presence of G51D fibril seeds.

\section{Discussion}

Structural polymorphs have been widely observed in amyloid fibrils formed by amyloid proteins such as $\alpha$-syn, Tau, and $A \beta^{18,32-36}$. Different fibril polymorphs exhibit distinct physiochemical and pathological properties, which are believed to underlie the clinicopathological variability of the associated diseases ${ }^{17,19,37-39}$. In this study, we found that the PD familial mutation G51D can trigger $\alpha$-syn to form a new fold assembling into an unusual right-handed fibril in PB buffer $(50 \mathrm{mM}$ phosphate buffer, pH 7.0, $50 \mathrm{mM} \mathrm{NaCl}$ ). The G51D fibril structure provides a structural explanation for how a single mutation G51D excludes the formation of other fibril strains (e.g. $\mathrm{WT}_{1 \mathrm{a}}$ and $\mathrm{E} 46 \mathrm{~K}$ strains) and induces the formation of a new strain. It is worthwhile to note that G51D $\alpha$-syn can also form polymorphic fibrils under different conditions. We previously observed that G51D a-syn can form a straight fibril in the Tris buffer containing $50 \mathrm{mM}$ Tris, pH 7.5, $150 \mathrm{mM} \mathrm{KCl}$, which is morphologically different from the left-handed twist fibril formed by WT $\alpha$-syn under the same condition (Supplementary Fig. 2) ${ }^{11}$. Unfortunately, due to the technical limitation of cryo-EM, we cannot yet solve untwisted fibril structures so far. Nevertheless, it will be interesting to investigate how G51D induces different fibril strains as well as their distinct pathological roles in disease.

In addition to determining the G51D fibril structure, we further demonstrate that WT $\alpha$-syn can also form the structure of the G51D fibril strain by cross-seeding. fPD patients with G51D mutation carry heterozygous SNCA missense mutation, which means that both WT and G51D $\alpha$-syn exist in the patients' brains. Since the G51D fibril can efficiently cross-seed WT $\alpha$-syn and induce it to form a G51D-like fibril structure, it is possible that the G51D strain may spread to the WT $\alpha$-syn and jointly accelerate the disease progression in fPD. In addition to the scenario of cross-seeding, it might also be biologically relevant to investigate whether WT and G51D $\alpha$-syn can co-aggregate to form amyloid fibrils. We thus mixed WT and G51D $\alpha$-syn monomers at a 1:1 molar ratio (termed as WT + G51D), and monitored the aggregation kinetics and fibril formation by ThT assay and NS-TEM. The result showed that mixing of WT and G51D a-syn generally enhanced the amyloid aggregation compared to individual proteins (Supplementary Fig. 7). Furthermore, mass spectrometry results confirmed that the WT + G51D fibrils indeed consist of both WT and G51D $\alpha$-syn (Supplementary Fig. 8). However, it is unclear whether WT and G51D proteins formed separate fibrils 
Table 1 Statistics of cryo-EM data collection and refinement.

\begin{tabular}{|c|c|c|c|}
\hline Name & G51D fibril & $\mathbf{W T}_{\text {51cs }}$ fibril & $\mathbf{W T}_{\text {wts }}$ fibril \\
\hline PDB & 7EOF & $\mathrm{n} / \mathrm{a}$ & $\mathrm{n} / \mathrm{a}$ \\
\hline EMDB & EMD-30931 & $\mathrm{n} / \mathrm{a}$ & $\mathrm{n} / \mathrm{a}$ \\
\hline \multicolumn{4}{|l|}{ Data collection } \\
\hline Magnification & 22,500 & 22,500 & 22,500 \\
\hline Pixel size $(\AA)$ & 1.06 & 1.06 & 1.06 \\
\hline Defocus range $(\mu \mathrm{m})$ & -1 to -2 & -1 to -2 & -1 to -2 \\
\hline Voltage (kV) & 300 & 300 & 300 \\
\hline Detector & $\mathrm{K} 3$ & K3 & K3 \\
\hline Microscope & Titan Krios & Titan Krios & Titan Krios \\
\hline Exposure time (s per frame) & 0.097 & 0.097 & 0.097 \\
\hline Number of frames & 32 & 32 & 32 \\
\hline Total dose $\left(e-\operatorname{per} \AA^{2}\right)$ & 55 & 55 & 55 \\
\hline \multicolumn{4}{|l|}{ Reconstruction } \\
\hline Micrographs & 1869 & 725 & 1056 \\
\hline Manually picked fibrils & 28,988 & 12,428 & 7903 \\
\hline Box size (pixel) & 288 & 686 & 686 \\
\hline Inter-box distance $(\AA)$ & 30.5 & 72.7 & 72.7 \\
\hline Initial particle images (no.) & 349,716 & 90,963 & 74,598 \\
\hline Final particle images (no.) & 213,348 & 40,587 & 16,120 \\
\hline Map resolution $(\AA)$ & 2.96 & $\mathrm{n} / \mathrm{a}$ & $\mathrm{n} / \mathrm{a}$ \\
\hline FSC threshold & 0.143 & $\mathrm{n} / \mathrm{a}$ & $\mathrm{n} / \mathrm{a}$ \\
\hline Map resolution $(\AA)$ & 3.15 & $\mathrm{n} / \mathrm{a}$ & $\mathrm{n} / \mathrm{a}$ \\
\hline FSC threshold & 0.5 & $\mathrm{n} / \mathrm{a}$ & $\mathrm{n} / \mathrm{a}$ \\
\hline $\begin{array}{l}\text { Map sharpening B-factor } \\
\left(\AA^{2}\right)\end{array}$ & -144.573 & $\mathrm{n} / \mathrm{a}$ & $\mathrm{n} / \mathrm{a}$ \\
\hline Helical rise $(\AA)$ & 2.43 & $\mathrm{n} / \mathrm{a}$ & $\mathrm{n} / \mathrm{a}$ \\
\hline Helical twist $\left({ }^{\circ}\right)$ & -179.37 & $\mathrm{n} / \mathrm{a}$ & $\mathrm{n} / \mathrm{a}$ \\
\hline \multicolumn{4}{|l|}{ Refinement } \\
\hline Initial model used & $6 \mathrm{~L} 4 \mathrm{~S}$ & $\mathrm{n} / \mathrm{a}$ & $\mathrm{n} / \mathrm{a}$ \\
\hline non-hydrogen atoms & 2,022 & $\mathrm{n} / \mathrm{a}$ & $\mathrm{n} / \mathrm{a}$ \\
\hline Protein residues & 294 & $\mathrm{n} / \mathrm{a}$ & $\mathrm{n} / \mathrm{a}$ \\
\hline Ligands & 0 & $\mathrm{n} / \mathrm{a}$ & $\mathrm{n} / \mathrm{a}$ \\
\hline r.m.s.d. bond lengths & 0.016 & $\mathrm{n} / \mathrm{a}$ & $\mathrm{n} / \mathrm{a}$ \\
\hline r.m.s.d. bond angles & 0.986 & $\mathrm{n} / \mathrm{a}$ & $\mathrm{n} / \mathrm{a}$ \\
\hline All-atom clash score & 9.76 & $\mathrm{n} / \mathrm{a}$ & $\mathrm{n} / \mathrm{a}$ \\
\hline Rotamer outliers & $0.00 \%$ & $\mathrm{n} / \mathrm{a}$ & $\mathrm{n} / \mathrm{a}$ \\
\hline Ramachandran outliers & $0.00 \%$ & $\mathrm{n} / \mathrm{a}$ & $\mathrm{n} / \mathrm{a}$ \\
\hline Ramachandran allowed & $12.77 \%$ & $\mathrm{n} / \mathrm{a}$ & $\mathrm{n} / \mathrm{a}$ \\
\hline Ramachandran favored & $87.23 \%$ & $\mathrm{n} / \mathrm{a}$ & $\mathrm{n} / \mathrm{a}$ \\
\hline
\end{tabular}

or hybrid fibrils. Future structure determination on WT + G51D fibrils may provide an answer. Moreover, other PD familial mutations such as E46K and A53T have been reported to induce the formation of distinct $\alpha$-syn fibril strains ${ }^{23-25}$. Further study is required to systemically investigate the cross-seeding and coaggregation activities and their mutual influences in fibril strain selection and $\alpha$-syn pathology under disease conditions.

\section{Methods}

Purification of recombinant WT and G51D $\alpha$-syn. Preparation of full-length WT and G51D $\alpha$-syn follows the similar protocol described previously ${ }^{11}$. Briefly, the genes encoding WT and G51D $\alpha$-syn were inserted into the pET22 vector. G51D point mutation gene was generated based WT $\alpha$-syn gene by PCR kit (TransGen Biotech, Cat. \# AP101-11). Primer sequences used in this work are listed in Supplementary Table 1. $\alpha$-Syn was co-expressed with yeast $\mathrm{N}$-acetyltransferase complex B in BL21 (DE3) cells (TransGen Biotech, Cat. \# CD601-02) to obtain the N-terminally acetylated $\alpha$-syn protein ${ }^{40}$. Cells were harvested after protein expression at $37^{\circ} \mathrm{C}$ for $4 \mathrm{~h}$ with $1 \mathrm{mM}$ isopropyl-1-thio-D-galactopyranoside (IPTG). Then, cells were lysed by sonication in $50 \mathrm{mM}$ Tris- $\mathrm{HCl}, \mathrm{pH} 8.0,1 \mathrm{mM}$ phenylmethylsulfonyl fluoride, $1 \mathrm{mM}$ EDTA. The supernatant was obtained by centrifugation at $15,000 \times g$ for $25 \mathrm{~min}$. Then the supernatant was processed by boiling at $100^{\circ} \mathrm{C}$ for $10 \mathrm{~min}$, streptomycin treatment $(20 \mathrm{mg} / \mathrm{ml}), \mathrm{pH}$ adjustment to 3.5 using $2 \mathrm{M} \mathrm{HCl}$, and dialysis $50 \mathrm{mM}$ Tris- $\mathrm{HCl}(\mathrm{pH} 8.0)$ overnight in turn. Anion exchange column (GE Healthcare, 17-5156-01) and Superdex 75 (GE Healthcare, 28-9893-33) were then used to purify $\alpha$-syn protein in high purity. For the anion exchange column, buffer $(50 \mathrm{mM}$ Tris-HCl, $1 \mathrm{M} \mathrm{NaCl}$, pH 8.0) was used to elute protein with a gradient $(0-60 \%)$.
Finally, an online EASY-nL-LC 1000 coupled with an Orbitrap Q-Exactive HF mass spectrometer was used to validate that the $\alpha$-syn protein is indeed acetylated.

Preparation of $\mathbf{W T}_{\mathbf{1 a}}$ and G51D $\boldsymbol{\alpha}$-syn fibril strains. Recombinant WT (100 $\mu \mathrm{M}$, in $50 \mathrm{mM}$ Tris, $\mathrm{pH} 7.5,150 \mathrm{mM} \mathrm{KCl}, 0.05 \% \mathrm{NaN}_{3}$ buffer $)$ and G51D $(100 \mu \mathrm{M}$, in $50 \mathrm{mM}$ phosphate buffer, $\mathrm{pH} 7.0,50 \mathrm{mM} \mathrm{NaCl}, 0.05 \% \mathrm{NaN}_{3}$ buffer) were shaking at $37^{\circ} \mathrm{C}, 900 \mathrm{rpm}$ in ThermoMixer (Eppendorf) for 7 days, respectively. $\alpha$-Syn preformed fibril seeds (PFFs) were obtained by sonication with $20 \%$ power for 15 times (1 s per time, $1 \mathrm{~s}$ per interval) on ice by JY92-IIN sonicator. Then, $100 \mu \mathrm{M}$ WT and G51D $\alpha$-syn monomer were incubated in the presence of $\alpha$-syn PFFs $\left(0.5 \mathrm{~mol} \%\right.$, concentration by monomer equivalent) at $900 \mathrm{rpm}, 37^{\circ} \mathrm{C}$ for a week. The residual soluble $\alpha$-syn in the supernatant was removed after pelleting the fibrils. The pellets were suspended with buffer to $100 \mu \mathrm{M}$ (equivalent to monomer concentration). The fibril samples were further used for NS-TEM, AFM, cryo-EM sample preparation, PK assay, sonication, and primary neuron treatment.

ThT kinetic assay. Seeding of WT and G51D monomer by a-syn PFFs was conducted by using ThT assay. $50 \mu \mathrm{M} \alpha$-syn WT (in $50 \mathrm{mM}$ Tris, pH 7.5, $150 \mathrm{mM}$ $\mathrm{KCl}, 0.05 \% \mathrm{NaN}_{3}$ ) monomer was incubated with the $\mathrm{WT}_{1 \mathrm{a}} \alpha$-syn PFFs and G51D a-syn PFFs ( $5 \mathrm{~mol} \%$, equivalent to monomer concentration) with $10 \mu \mathrm{M}$ ThT in the reaction mixture, separately. Similarly, $50 \mu \mathrm{M} \alpha$-syn G51D (in $50 \mathrm{mM}$ phosphate buffer, $\mathrm{pH} 7.0,50 \mathrm{mM} \mathrm{NaCl}, 0.05 \% \mathrm{NaN}_{3}$ ) monomer was incubated with the G51D ( $5 \mathrm{~mol} \%$, equivalent to monomer concentration) with $10 \mu \mathrm{M}$ ThT in the reaction mixture. A Fluoroskan Ascent microplate reader (Thermo Scientific) was used to test reactions performed in a 384-well optical plate (Thermo Scientific) in triplicate, with $440 \mathrm{~nm}$ excitation wave-length and $485 \mathrm{~nm}$ emission wave-length, a bottom read. Graphing was performed with GraphPad Prism 6. The data shown in each ThT experiment are mean \pm s.d., $n=5$ independent samples.

A co-aggregation experiment of $\alpha$-syn WT and G51D was conducted by using ThT assay. $100 \mu \mathrm{M} a$-syn WT, $100 \mu \mathrm{M}$ G51D monomer or mixed monomer $(50 \mu \mathrm{M}$ WT $+50 \mu \mathrm{M}$ G51D) was incubated with $10 \mu \mathrm{M}$ ThT in the reaction mixture in PB buffer ( $50 \mathrm{mM}$ phosphate buffer, $\mathrm{pH} 7.0,50 \mathrm{mM} \mathrm{NaCl}, 0.05 \% \mathrm{NaN}_{3}$ ) or Tris Buffer $\left(50 \mathrm{mM}\right.$ Tris, $\left.\mathrm{pH} 7.5,150 \mathrm{mM} \mathrm{KCl}, 0.05 \% \mathrm{NaN}_{3}\right)$. A Fluoroskan Ascent microplate reader (Thermo Scientific) was used to test reactions performed in a 384-well optical plate (Thermo Scientific) in triplicates, with $440 \mathrm{~nm}$ excitation wavelength and $485 \mathrm{~nm}$ emission wavelength, a bottom read. Graphing was performed with GraphPad Prism 6. The data shown in each ThT experiment are mean \pm s.d., $n=3$ independent samples.

The sample from the ThT assay was further characterized by NS-TEM. $50 \mu \mathrm{l}$ aqueous solution of each sample from the ThT assay was pelleted by centrifugation $\left(14,462 \times g, 25^{\circ} \mathrm{C}, 45 \mathrm{~min}\right)$. SDS-loading buffer was added into $45 \mu \mathrm{l}$ supernatant and boiled for $10 \mathrm{~min}$. The pellet was washed by phosphate-buffered saline (PBS) and dissolved in $45 \mu \mathrm{l}$ buffer, then sonicated for $5 \mathrm{~min}$ and boiled for $30 \mathrm{~min}$. Lastly, the solution was boiled in the SDS-loading buffer for $10 \mathrm{~min}$. The supernatant samples and dissolved pellet samples were loaded on $4-20 \%$ Bis-Tris gels (GenScript), respectively. The gels were stained by Coomassie brilliant blue and images were acquired and analyzed with Image Lab 3.0 (Bio-Rad).

Atomic force microscopy. Fibril samples were mounted on mica for $3 \mathrm{~min}$, rinsed gently with water, and dried with nitrogen flow. Images were captured by Nanoscope V Multimode 8 (Bruker) with SNL-10 probes (a constant of $0.35 \mathrm{~N} \mathrm{~m}^{-1}$ ) on ScanAsyst air mode. Images were acquired with a $1.5 \mathrm{~Hz}$ rate at $512 \times 512$ pixels and analyzed on the Nanoscope software.

Negative-staining transmission electron microscopy. Samples were prepared by loading $5 \mu$ l of fibril solution onto a glow-discharged 200 mesh carbon support film (Zhongjingkeyi Technology Co., Ltd., Beijing). The samples were held for $45 \mathrm{~s}$ and washed with double-distilled water followed by $3 \%$ uranyl acetate. The grid was then stained with $3 \%$ uranyl acetate for $45 \mathrm{~s}$ and allowed to dry in air. The samples were imaged by a Tecnai T12 microscope (FEI).

PK digestion of $\boldsymbol{\alpha}$-syn PFFs. $\mathrm{WT}_{1 \mathrm{a}}$ or G51D $\alpha$-syn PFFs were prepared by sonication at $20 \%$ power for 15 times (1 s per time, $1 \mathrm{~s}$ per interval) on ice by JY92-IIN sonicator. $\alpha$-Syn PFFs $\left(3 \mathrm{mg} \mathrm{ml}^{-1}, 25 \mu \mathrm{l}\right.$, in PBS, $\mathrm{pH}$ 7.4) were incubated with proteinase $\mathrm{K}$ (final concentration 0.5 and $1.5 \mu \mathrm{g} \mathrm{ml}-1$, Invitrogen) at $37^{\circ} \mathrm{C}$ for $10,30,60$ and $200 \mathrm{~min} .1 \mathrm{mM}$ PMSF was added to samples to stop the reaction. Then samples were boiled with an SDS-loading buffer for $15 \mathrm{~min}$ and loaded on 4-20\% Bis-Tris gels (GenScript). The gels were stained by Coomassie brilliant blue and images were recorded and analyzed with Image Lab 3.0 (Bio-Rad). Graphing was performed with GraphPad Prism 6. The data shown in are mean \pm s.d., $n=3$ independent samples.

Characterization of fibril fragmentation. $20 \mu \mathrm{M} \mathrm{WT}_{1 \mathrm{a}}$ or $\mathrm{G} 51 \mathrm{D} \alpha$-syn fibrils were sonicated with $20 \%$ power 2 times and 15 times on ice by JY92-IIN sonicator. And $5 \mu \mathrm{M} \mathrm{WT}$ 1a or G51D $\alpha$-syn PFFs (under the corresponding condition) were characterized by NS-TEM.

Cell viability assay. SH-SY5Y cells were cultured to test the cytotoxicity of G51D and WT1a PFFs with a CCK-8 kit. SH-SY5Y cells cultured in a 96-well plate were 
a

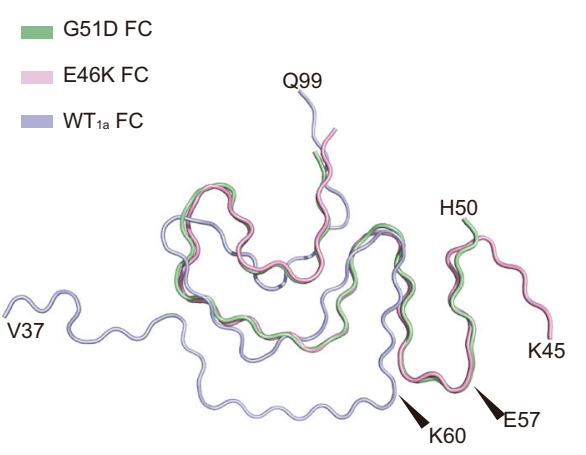

b

b $\quad 99 \xi$
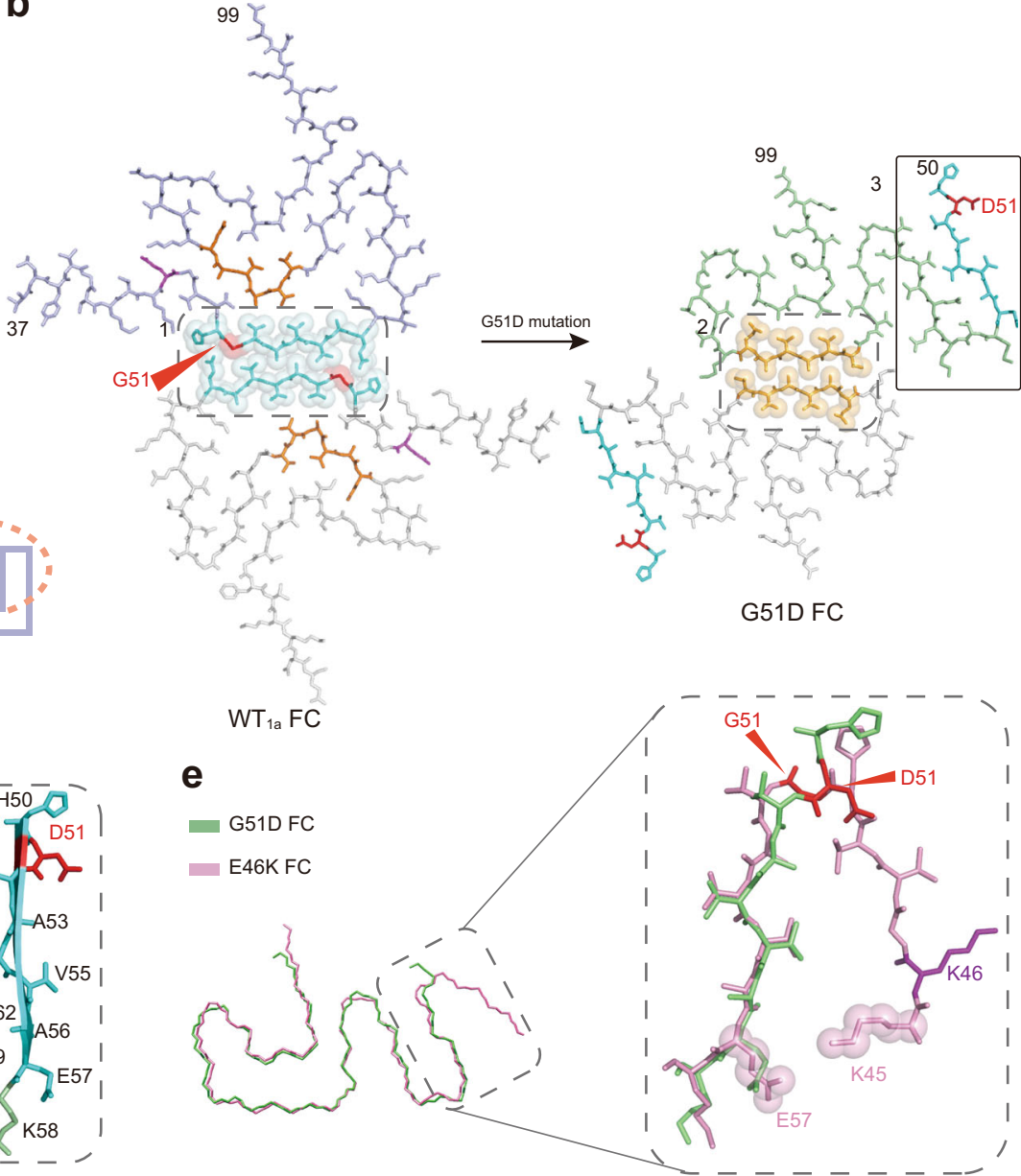

Fig. 3 Structure comparison of G51D, $\mathbf{W T}_{\mathbf{1 a}}$, and E46K fibrils. a Overlay of the structures of every single $\alpha$-syn subunit from the G51D, E46K, and WT fibrils. G51D fibril is in green; $\mathrm{WT}_{1 a}$ fibril is in slate; E46K fibril is in pink. The region with a similar structure shared by three different FC is circled on topology diagrams. b Rearrangement of $\alpha$-syn fibril structure triggered by G51D mutation. Residues involved in the protofilament interfaces are presented in spheres. The protofilament interface of $\mathrm{WT}_{1 \mathrm{a}}$ fibril is colored in cyan; the interface of the G51D fibril is colored in orange. D51 and G51 are highlighted in red. E46 and K46 are in purple. c Zoom-in views of the WT $_{1 a}$ and G51D fibril interfaces. $\mathbf{d}$ The $\beta$-hairpin (residues 50-66) formed in the G51D fibril structure is zoomed in with D51 in red. e Overlay of the structures of every single $\alpha$-syn subunit from the G51D and E46K fibrils. G51D mutation disrupts the $\beta$-turn formed of residues 45-57 formed in the E46K fibril. G51 and D51 are highlighted in red. K46 is highlighted in purple.

treated with $\alpha$-syn PFFs at final concentrations of $0.01,0.1$, and $1 \mu \mathrm{M}$ for $24 \mathrm{~h}$. Then cell viability was tested with the CCK-8 kit following the manufacturer's protocol. Briefly, CCK-8 solution $(10 \mu \mathrm{l} /$ well) was added to each well. The absorbance of the plate was measured at $450 \mathrm{~nm}$ after incubating for $30 \mathrm{~min}$. The data were analyzed with GraphPad Prism 6.

Primary neuronal culture experiments. Primary cortical neurons were dissected from the cortex of embryonic day (E) 16-E18 Sprague Dawley rats (Shanghai SIPPR BK Laboratory Animals Ltd, China) embryos as previously described ${ }^{41}$. In brief, primary neurons were seeded onto coverslips previously coated with poly-Dlysine (PDL) in 24-well plates (150,000 cells/coverslip). At 8-day in vitro (DIV), neurons were treated with PBS and $100 \mathrm{nM}$ (final concentration) $\mathrm{WT}_{1 \mathrm{a}}$ or G51D $\alpha$ syn PFFs, and collected for immunofluorescence at 10/14-day post-treatment. The primary antibodies used in the assay were the phospho- $\alpha$-synuclein (S129) antibody (Abcam, cat. no. ab51253) and the MAP2 antibody (Abcam, cat. no. ab5392). The antibodies were diluted at a ratio of 1:1000. The intensity of confocal images was analyzed by Image J 2.0.0. The secondary antibodies included goat anti-rabbit IgG Alexa Fluor 568 (Abcam, cat. no. ab175471) and goat anti-chicken Alexa Fluor 488 (Thermo Fisher, cat. no. A-11039). All rat experiments were performed followed the protocols approved by the Animal Care Committee of the Interdisciplinary Research Center on Biology and Chemistry (IRCBC), Chinese Academy of Sciences (CAS). There are three samples in each group.

Purity characterization of G51D fibril. To characterize the purity of $\alpha$-syn fibrils, we dissolved the fibrils with buffer (50 mM Tris, pH 8.0, $150 \mathrm{mM} \mathrm{NaCl}, 1 \%$ Triton $\mathrm{X}-100,2 \%$ SDS) and performed SDS-PAGE. The gels were stained by Coomassie brilliant blue and images were acquired and analyzed with Image Lab 3.0 (Bio-Rad) In addition, the fibrils were also detected by western blot. The monomer and fibrils were immunoblotted by anti- $\alpha$-synuclein antibodies: $2642 \mathrm{~S}$ (Cell signaling) and ab138501 (Abcam), respectively. The antibodies were diluted at a ratio of 1:1000.

MALDI-TOF MS. A droplet of $1 \mu$ l solution containing G51D monomer or dissolved fibrils was mixed with $1 \mu \mathrm{l}$ of the sinapinic acid matrix ( $10 \mathrm{mg}$ of sinapinic acid per $\mathrm{ml}$, a $70: 30$ water/acetonitrile (ACN) with $0.1 \%$ trifluoroacetic acid).

Deposit $1 \mu \mathrm{l}$ of final mix onto a MALDI stainless steel target and allow to air dry at room temperature. A 5800 MALDI-TOF/TOF mass spectrometer (AB SCIEX, Framingham, MA, USA) in linear positive mode with a mass range from $\mathrm{m} / \mathrm{z} 2500$ to 20,000 was used for MALDI-TOF MS measurements and analyses.

LC-MS/MS analysis. Excise bands of dissolved fibril samples loaded on $4-20 \%$ Bis-Tris gels with a clean scalpel and cuts gel bands into cubes. Gel pieces were then destained with ammonium bicarbonate/ACN $(1: 1, \mathrm{vol} / \mathrm{vol})$, shrink with neat $\mathrm{ACN}$, and then saturated gel pieces with trypsin. After digestion with trypsin by incubating samples overnight at $37^{\circ} \mathrm{C}$, extract peptide digestion products by incubating samples with extraction buffer (1:2 (vol/vol) $5 \%$ formic acid (FA)/ACN for $15 \mathrm{~min}$ at $37^{\circ} \mathrm{C}$ in a shaker. After centrifugation at $16,000 \times g$ and $4{ }^{\circ} \mathrm{C}$ for $15 \mathrm{~min}$, the clear supernatants were collected, dry down in a vacuum centrifuge, and then resuspended in $0.1 \%$ (vol/vol) FA for further LC-MS/MS analysis.

The peptide mixture was analyzed using an Orbitrap Fusion mass spectrometer coupled to an online EASY-nL-LC 1000 system. Mobile phase A consisted of $0.1 \%$ FA, $2 \% \mathrm{ACN}$, and $98 \% \mathrm{H}_{2} \mathrm{O}$, and mobile phase B consisted of $0.1 \% \mathrm{FA}, 2 \% \mathrm{H}_{2} \mathrm{O}$, and $98 \%$ ACN. A 60 min gradient (mobile phase B: $3 \%$ at $0 \mathrm{~min}, 8 \%$ at $5 \mathrm{~min}, 20 \%$ at 

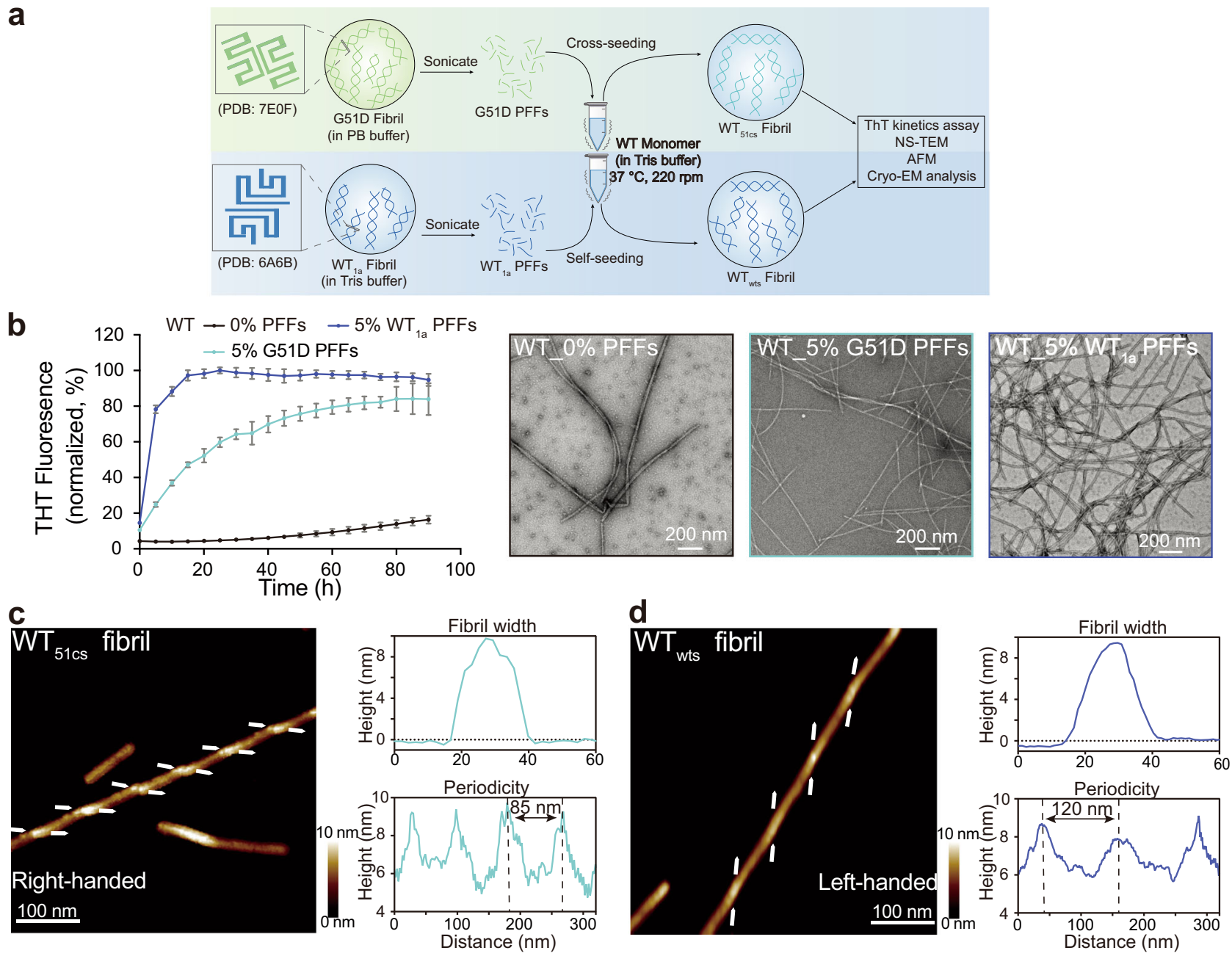

Fig. 4 Seeding of WT $\boldsymbol{\alpha}$-syn by both G51D and WT $\boldsymbol{\alpha}$-syn PFFs. a Schematic diagram of the seeding process. PDB IDs of WT 1 and G51D fibrils used for the seeding experiments are provided in parentheses. Buffer $\left(50 \mathrm{mM}\right.$ Tris, $\mathrm{pH} 7.5,150 \mathrm{mM} \mathrm{KCl}, 0.05 \% \mathrm{NaN}_{3}$ ) is referred to as Tris buffer. Buffer ( $50 \mathrm{mM}$ phosphate buffer, $\mathrm{pH} 7.0,50 \mathrm{mM} \mathrm{NaCl}, 0.05 \% \mathrm{NaN}_{3}$ buffer) is referred to as PB buffer. The schematic diagram was created from scratch. $\mathbf{b}$ Fibrillation of WT $\alpha$-syn alone (colored in black) or in the presence of 5 mol\% WT PFFs (colored in purple) and G51D PFFs (colored in cyan), respectively. Data are shown as mean \pm s.d., $n=5$ independent samples. The fibrils sampled at the end of the ThT kinetics assay were imaged by NS-TEM (right panel). Scale bar: $200 \mathrm{~nm}$. c and d AFM images of the $\mathrm{WT}_{51 \mathrm{cs}}$ (c) and $\mathrm{WT}_{\text {wts }}$ fibrils (d). The images represent reproducible results in three independent experiments. The arrows at both sides of the fibril indicate the starting points of the fibril protrusions to clarify the handedness. Images were acquired with a $1.45 \mathrm{~Hz}$ rate at $512 \times 512$ pixels. The fibril width and periodicity were analyzed by the Nanoscope software.

$46 \mathrm{~min}, 30 \%$ at $54 \mathrm{~min}, 95 \%$ at $55 \mathrm{~min}$, and $95 \%$ at $60 \mathrm{~min}$ ) was used at a static flow rate of $300 \mathrm{~nL} / \mathrm{min}$. The data were acquired in a data-dependent (top 20) mode. Highenergy collisional dissociation (HCD) was used to fragment the precursor peptides, and the resulting fragment ions were measured in the ion trap analyzer.

Cryo-EM data collection. A solution containing G51D or $\mathrm{WT}_{51 \mathrm{cs}}$ fibril samples was applied to glow-discharged holey carbon Cu Quantifoil grids (R2/1, 300 mesh) and then plunge-frozen in liquid ethane after blotting with filter paper using Vitrobot Mark IV(FEI). Cryo-EM micrographs with a defocus from -1 to $-2 \mu \mathrm{m}$ were collected on a Gatan K3 direct detector in super-resolution mode on a Titan Krios transmission electron microscope (FEI) operated at $300 \mathrm{kV} .32$ movies were recorded per micrograph was a record with a pixel size of $1.06 \AA$ pixel $^{-1}$ using a dose of $55 \mathrm{e}^{-} \AA^{-2}$. Automated cryo-EM data collection was performed by Serial EM software ${ }^{42}$.

Imaging processing, reconstruction, and model building. MotionCorr $2^{43}$ was used to correct beam-induced motion of movie frames with dose weighting while CTFFIND4.1.8 ${ }^{44}$ was used to estimate the contrast transfer function. All filaments were picked manually using the manual picking method of RELION3.0 ${ }^{45}$. All subsequent steps of helical reconstruction were carried out using RELION 3.0.

G51D dataset. 28,989 filaments were manually picked from 1869 micrographs. G51D segments were first extracted using 1024-pixel box size with an inter-box distance of $109 \AA$ and used for subsequent reference-free $2 \mathrm{D}$ classification with a decreasing in-plane angular sampling rate from $12^{\circ}$ to $1^{\circ}$ and a $T=2$ regularization parameter to estimate the fibril pitch and helical parameters. Filaments containing selected segments with 1024-pixel box size were then re-extracted using 288-pixel box size with an inter-box distance of $31 \AA$ and particles comprising an entire helical crossover were selected for the following $3 \mathrm{D}$ classification. An initial $3 \mathrm{D}$ reference was de novo generated using selected particles after 2D classification by relion_helix_inimodel $2 \mathrm{~d}$ and the initial 3D model low-pass filtered to $60 \AA$ was then applied further as a reference map to perform 3D classification. Local optimization of helical twist and rise was performed while $\beta$-strands perpendicular to the helical axis was clearly separated. The symmetry of psedo- $2_{1}$ was applied and several rounds of $3 \mathrm{D}$ classification with $K=3$ and $K=1$ were used to gain segments belonging to the same conformation and optimize twist parameters. Optimized parameters and selected segments were applied for high-resolution gold-standard refinement. Post-processing with a soft-edge solvent mask in $30 \%$ central $\mathrm{Z}$ length was performed to sharpen the refined maps. The final overall resolution estimate was calculated to be $2.96 \AA$ based on the 0.143 Fourier shell correlation cutoff. The model was built into the central region of the sharpened density map using E46K $\alpha$-syn structure ((PDB entry code $6 \mathrm{~L} 4 \mathrm{~S})$ as an initial model in $\mathrm{COOT}^{46}$. A three-layer model was generated and refined by the real_space_refine program in PHENIX ${ }^{47,48}$.

WT $_{\text {51cs }}$ dataset. 12,428 filaments were manually picked from 725 micrographs. $\mathrm{WT}_{51 \mathrm{cs}}$ segments were first extracted using 1200-pixel box size with an inter-box distance of $127 \AA$ and used for several iterations of reference-free 2D classification. 

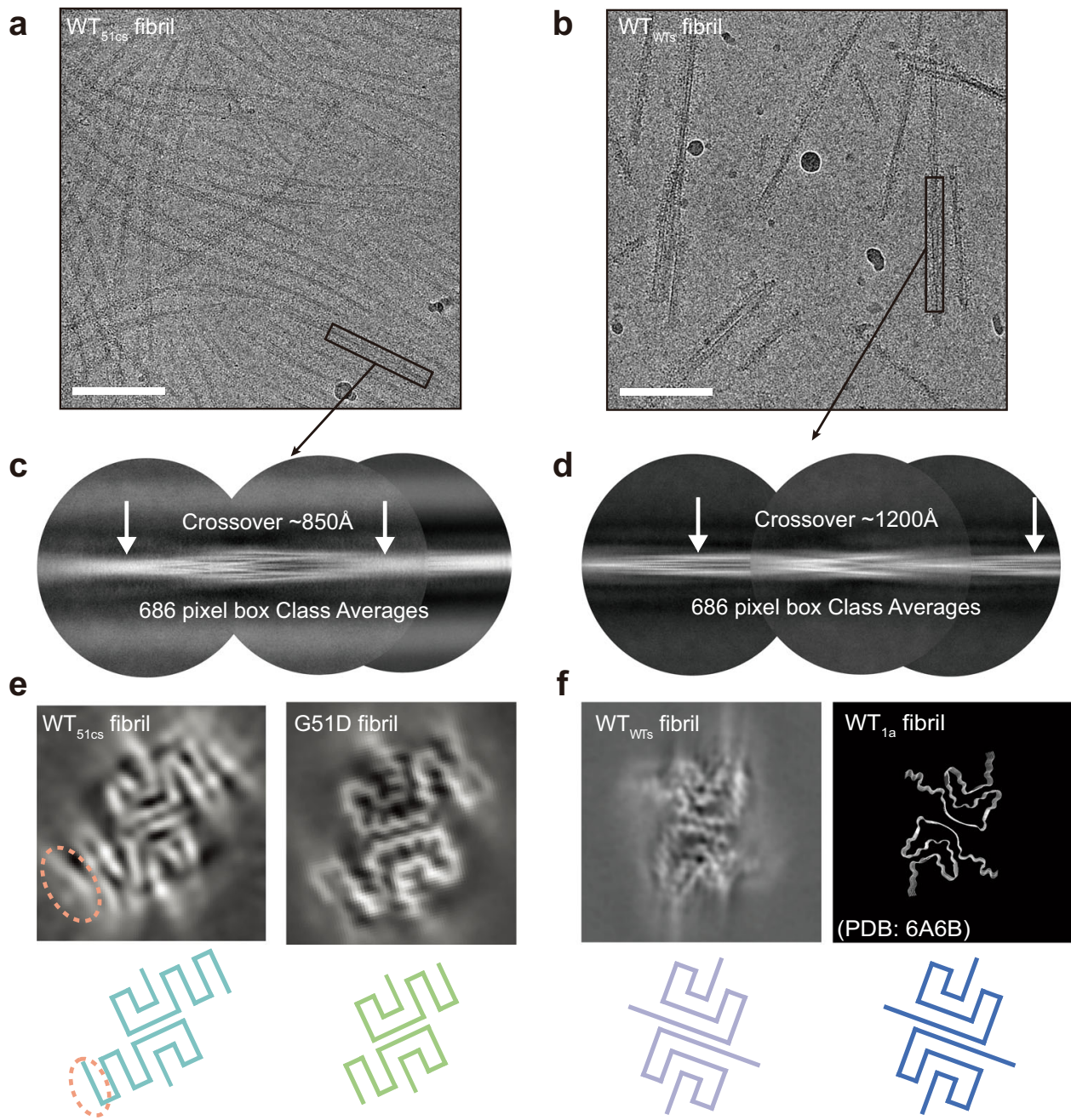

Fig. 5 Cryo-EM study of $\mathbf{W T}_{\text {51cs }}$ and $\mathbf{W T}_{\text {wts }}$ strains. Cryo-EM micrographs of $\mathrm{WT}_{51 c s}$ strain (a) and $\mathrm{WT}_{\text {wts }}$ strain (b) are shown. The images represent reproducible results in three independent experiments. Scale bar $=100 \mathrm{~nm}$. 686-pixel box size 2D class averages comprising a helical crossover of $\mathrm{WT}_{51 \mathrm{cs}}$ strain (c) and $\mathrm{WT}_{\text {wts }}$ strain (d) are shown and used to determine crossover distance. e Central slice of the 3D map of the WT $\mathrm{W}_{51 \mathrm{cs}}$ and G51D strains. The additional electron density of the $\mathrm{WT}_{51 \mathrm{cs}}$ strain compared to the G51D strain is circled. $\mathbf{f}$ Central slice of the 3D map of the WT $\mathrm{Wts}_{\text {wts }}$ (left). Structural models of $\mathrm{WT}_{1 \mathrm{a}}$ strain (PDB ID: 6A6B) are shown on the right. The topology diagram of $\alpha$-syn for each strain is shown (bottom).

Filaments containing selected segments were then re-extracted using a 686-pixel box size with an inter-box distance of $73 \AA$ and used for subsequent reference-free 2D classification. An initial 3D reference was de novo generated by relion_helix_inimodel2d using selected particles comprising an entire helical crossover and the initial 3D model low-pass filtered to $60 \AA$ was then applied further as a reference map to perform de novo 3D classification. Local optimization of helical twist and rise was performed while $\beta$-strands perpendicular to the helical axis was clearly separated. Several rounds of 3D classification with $K=3$ and $K=1$ were then performed.

WT $_{\text {wts }}$ dataset. 7903 filaments were manually picked from 1056 micrographs and first extracted with a 1024-pixel box size with an inter-box distance of $109 \AA$ and used for several iterations of reference-free 2D classification. Filaments containing selected segments were then re-extracted using a 686-pixel box size with an inter-box distance of $73 \AA$ A. Similarly, with G51D and $W^{2} T_{51 c s}$ dataset, an initial $3 \mathrm{D}$ reference was de novo generated by relion helix inimodel $2 \mathrm{~d}$ using selected particles comprising an entire helical crossover, and the initial 3D model low-pass filtered to $60 \AA$ was then applied further as a reference map to perform de novo 3D classification. Several rounds of 3D classification with $K=3$ and $K=1$ were then performed.

Reporting summary. Further information on experimental design is available in the Nature Research Reporting Summary linked to this paper.

\section{Data availability}

Density maps of the G51D fibril are available through EMDB with entry code: EMD30931. The structural model was deposited in the Protein Data Bank with entry code:
7E0F. Other structural models used in this study are available in the Protein Data Bank with entry codes: 6A6B ( $\alpha$-synuclein polymorph 1a fibril), 6L4S (E46K a-synuclein fibril). The source data underlying Figs. 1a, b, c, and 4b, c, d, Supplementary Figs. 1a, b, c, $3 a, 4 a, c$, and $6 a, b$ are provided as a Source Data file with this paper. Other data that support the findings of this study are available from the corresponding author upon reasonable request. Source data are provided with this paper.

Received: 1 May 2021; Accepted: 7 October 2021;

Published online: 29 October 2021

\section{References}

1. Spillantini, M. G. et al. a-Synuclein in Lewy bodies. Nature 388, 839-840 (1997).

2. Spillantini, M. G. \& Goedert, M. The a-synucleinopathies: Parkinson's disease, dementia with Lewy bodies, and multiple system atrophy. Ann. N. Y. Acad. Sci. 920, 16-27 (2000)

3. Woerman, A. L. et al. a-Synuclein: multiple system atrophy prions. Cold Spring Harb. Perspect. Med. 8, a024588 (2018).

4. Olanow, C. W. \& Prusiner, S. B. Is Parkinson's disease a prion disorder? Proc Natl Acad. Sci. USA 106, 12571-12572 (2009).

5. Luk, K. C. et al. Pathological $\alpha$-synuclein transmission initiates Parkinson-like neurodegeneration in nontransgenic mice. Science 338, 949-953 (2012).

6. Soto, C. \& Pritzkow, S. Protein misfolding, aggregation, and conformational strains in neurodegenerative diseases. Nat. Neurosci. 21, 1332-1340 (2018). 
7. Kim, S. et al. Transneuronal propagation of pathologic $\alpha$-synuclein from the gut to the brain models Parkinson's disease. Neuron 103, 627-641 (2019).

8. Tuttle, M. D. et al. Solid-state NMR structure of a pathogenic fibril of fulllength human a-synuclein. Nat. Struct. Mol. Biol. 23, 409-415 (2016).

9. Guerrero-Ferreira, R. et al. Cryo-EM structure of alpha-synuclein fibrils. Elife 7, e36402 (2018)

10. Li, B. et al. Cryo-EM of full-length $\alpha$-synuclein reveals fibril polymorphs with a common structural kernel. Nat. Commun. 9, 1-10 (2018).

11. Li, Y. et al. Amyloid fibril structure of $\alpha$-synuclein determined by cryoelectron microscopy. Cell Res. 28, 897-903 (2018).

12. Guerrero-Ferreira, R. et al. Two new polymorphic structures of human fulllength alpha-synuclein fibrils solved by cryo-electron microscopy. Elife $\mathbf{8}$, e48907 (2019).

13. Ni, X., McGlinchey, R. P., Jiang, J. \& Lee, J. C. Structural insights into asynuclein fibril polymorphism: effects of Parkinson's disease-related Cterminal truncations. J. Mol. Biol. 431, 3913-3919 (2019).

14. Schweighauser, M. et al. Structures of $\alpha$-synuclein filaments from multiple system atrophy. Nature 585, 464-469 (2020).

15. Prusiner, S. B. et al. Evidence for $\alpha$-synuclein prions causing multiple system atrophy in humans with parkinsonism. Proc. Natl Acad. Sci. USA 112, E5308-E5317 (2015).

16. Alafuzoff, I. \& Hartikainen, P. Alpha-synucleinopathies. Handb. Clin. Neurol. 145, 339-353 (2018)

17. Peng, C. et al. Cellular milieu imparts distinct pathological $\alpha$-synuclein strains in a-synucleinopathies. Nature 557, 558-563 (2018).

18. Strohäker, T. et al. Structural heterogeneity of $\alpha$-synuclein fibrils amplified from patient brain extracts. Nat. Commun. 10, 1-12 (2019).

19. Peelaerts, W. et al. $\alpha$-Synuclein strains cause distinct synucleinopathies after local and systemic administration. Nature 522, 340-344 (2015).

20. Van der Perren, A. et al. The structural differences between patient-derived $\alpha$ synuclein strains dictate characteristics of Parkinson's disease, multiple system atrophy and dementia with Lewy bodies. Acta Neuropathol. 139, 977-1000 (2020).

21. Shahnawaz, M. et al. Discriminating a-synuclein strains in Parkinson's disease and multiple system atrophy. Nature 578, 273-277 (2020).

22. Boyer, D. R. et al. Structures of fibrils formed by a-synuclein hereditary disease mutant H50Q reveal new polymorphs. Nat. Struct. Mol. Biol. 26, 1044-1052 (2019).

23. Boyer, D. R. et al. The $\alpha$-synuclein hereditary mutation E46K unlocks a more stable, pathogenic fibril structure. Proc. Natl Acad. Sci. USA 117, 3592-3602 (2020).

24. Sun, Y. et al. Cryo-EM structure of full-length $\alpha$-synuclein amyloid fibril with Parkinson's disease familial A53T mutation. Cell Res. 30, 360-362 (2020).

25. Zhao, K. et al. Parkinson's disease associated mutation E46K of $\alpha$-synuclein triggers the formation of a distinct fibril structure. Nat. Commun. 11, 1-9 (2020).

26. Zhao, K. et al. Parkinson's disease-related phosphorylation at Tyr39 rearranges $\alpha$-synuclein amyloid fibril structure revealed by cryo-EM. Proc. Natl Acad. Sci. USA 117, 20305-20315 (2020).

27. Lesage, $\mathrm{S}$. et al. G51D $\alpha$-synuclein mutation causes a novel Parkinsonian-pyramidal syndrome. Ann. Neurol. 73, 459-471 (2013).

28. Kiely, A. P. et al. $\alpha$-Synucleinopathy associated with G51D SNCA mutation: a link between Parkinson's disease and multiple system atrophy? Acta Neuropathol. 125, 753-769 (2013).

29. Rutherford, N. J., Moore, B. D., Golde, T. E. \& Giasson, B. I. Divergent effects of the H50Q and G51D SNCA mutations on the aggregation of $\alpha$-synuclein. J. Neurochem. 131, 859-867 (2014).

30. Hayakawa, H. et al. Structurally distinct $\alpha$-synuclein fibrils induce robust Parkinsonian pathology. Mov. Disord. 35, 256-267 (2020).

31. Volpicelli-Daley, L. A., Luk, K. C. \& Lee, V. M. Y. Addition of exogenous asynuclein preformed fibrils to primary neuronal cultures to seed recruitment of endogenous $\alpha$-synuclein to Lewy body and Lewy neurite-like aggregates. Nat. Protoc. 9, 2135-2146 (2014)

32. Fitzpatrick, A. W. P. et al. Cryo-EM structures of tau filaments from Alzheimer's disease. Nature 547, 185-190 (2017).

33. Qiang, W., Yau, W.-M., Lu, J.-X., Collinge, J. \& Tycko, R. Structural variation in amyloid- $\beta$ fibrils from Alzheimer's disease clinical subtypes. Nature 541, 217-221 (2017).

34. Falcon, B. et al. Structures of filaments from Pick's disease reveal a novel tau protein fold. Nature 561, 137-140 (2018).

35. Falcon, B. et al. Novel tau filament fold in chronic traumatic encephalopathy encloses hydrophobic molecules. Nature 568, 420-423 (2019).

36. Zhang, W. et al. Novel tau filament fold in corticobasal degeneration. Nature 580, 283-287 (2020)

37. Nussbaum, R. L. \& Ellis, C. E. Alzheimer's disease and Parkinson's disease. $N$. Engl. J. Med. 348, 1356-1364 (2003).
38. Irwin, D. J., Lee, V. M. Y. \& Trojanowski, J. Q. Parkinson's disease dementia: convergence of $\alpha$-synuclein, tau and amyloid- $\beta$ pathologies. Nat. Rev. Neurosci. 14, 626-636 (2013).

39. Li, D. \& Liu, C. Hierarchical chemical determination of amyloid polymorphs in neurodegenerative disease. Nat. Chem. Biol. 17, 237-245 (2021).

40. Johnson, M., Coulton, A. T., Geeves, M. A. \& Mulvihill, D. P. Targeted aminoterminal acetylation of recombinant proteins in E. coli. PLoS ONE 5, el5801 (2010).

41. Volpicelli-Daley, L. A., Luk, K. C. \& Lee, V. M. Addition of exogenous alphasynuclein preformed fibrils to primary neuronal cultures to seed recruitment of endogenous alpha-synuclein to Lewy body and Lewy neurite-like aggregates. Nat. Protoc. 9, 2135-2146 (2014).

42. Mastronarde, D. N. Automated electron microscope tomography using robust prediction of specimen movements. J. Struct. Biol. 152, 36-51 (2005).

43. Zheng, S. Q. et al. MotionCor2: anisotropic correction of beam-induced motion for improved cryo-electron microscopy. Nat. Methods 14, 331-332 (2017).

44. Rohou, A. \& Grigorieff, N. CTFFIND4: fast and accurate defocus estimation from electron micrographs. J. Struct. Biol. 192, 216-221 (2015).

45. Zivanov, J. et al. New tools for automated high-resolution cryo-EM structure determination in RELION-3. eLife 7, https://doi.org/10.7554/ eLife.42166 (2018)

46. Emsley, P., Lohkamp, B., Scott, W. G. \& Cowtan, K. Features and development of Coot. Acta Crystallogr. Sect. D. 66, 486-501 (2010).

47. Afonine, P. V., Headd, J., Terwilliger, T. \& Adams, P. New tool: Phenix.realspace-refine. Comput. Crystallogr. Newsl. 4, 43-44 (2013).

48. Adams, P. D. et al. PHENIX: a comprehensive Python-based system for macromolecular structure solution. Acta Crystallogr. Sect. D. 66, 213-221 (2010).

\section{Acknowledgements}

This work was supported by the Major State Basic Research Development Program Grant No. 2019YFE0120600), the National Natural Science Foundation (NSF) of China (Grant No. 91853113 and 31872716), the Science and Technology Commission of Shanghai Municipality (Grant No. 18JC1420500), the Shanghai Municipal Science and Technology Major Project (Grant No. 2019SHZDZX02), the Shanghai Science and Technology Committee (Grant No. 20XD1425000), the CAS project for Young Scientists in Basic research (Grant No.YSBR-009), the Shanghai Pujiang Program (Grant No. 19PJ1405000). We would like to thank the Bio-Electron Microscopy Facility of ShanghaiTech University, and we are grateful to Qianqian Sun and Yaping Wang for their help in cryo-EM data collection. We thank the staff members of the Mass Spectrometry System at the National Facility for Protein Science in Shanghai (NFPS), Zhangjiang Lab, China, for providing technical support and assistance in MALDI-TOF MS data collection and analysis.

\section{Author contributions}

D.L. and C.L. designed the project. H.L. and Y.S. prepared the WT and G51D $\alpha$-syn fibril samples and performed an AFM assay. H.L. performed the biochemical assays. Y.S. prepared cryo-EM samples, performed cryo-EM data collection and processing. W.X., Q.C., X.Z. and B.S. assisted in cryo-EM data collection and processing. B.D. assisted in performing AFM assay. K.W. and Y.Z. performed LC-MS/MS data collection and analysis. Y.S. and H.L. prepared samples for MALDI-TOF MS. All the authors are involved in analyzing the data and contributed to manuscript discussion and editing. D.L. and C.L. wrote the manuscript.

\section{Competing interests}

The authors declare no competing interests.

\section{Additional information}

Supplementary information The online version contains supplementary material available at https://doi.org/10.1038/s41467-021-26433-2.

Correspondence and requests for materials should be addressed to Cong Liu.

Peer review information Nature Communications thanks Joon Won Park and the other anonymous, reviewer(s) for their contribution to the peer review of this work.

Reprints and permission information is available at http://www.nature.com/reprints

Publisher's note Springer Nature remains neutral with regard to jurisdictional claims in published maps and institutional affiliations. 
(c) (i) Open Access This article is licensed under a Creative Commons Attribution 4.0 International License, which permits use, sharing, adaptation, distribution and reproduction in any medium or format, as long as you give appropriate credit to the original author(s) and the source, provide a link to the Creative Commons license, and indicate if changes were made. The images or other third party material in this article are included in the article's Creative Commons license, unless indicated otherwise in a credit line to the material. If material is not included in the article's Creative Commons license and your intended use is not permitted by statutory regulation or exceeds the permitted use, you will need to obtain permission directly from the copyright holder. To view a copy of this license, visit http://creativecommons.org/ licenses/by/4.0/.

(C) The Author(s) 2021 